\title{
A NOTE \\ ON THE CONTINUED FRACTION OF MINKOWSKI
}

\author{
HENDRIK JAGER
}

\begin{abstract}
Denote by $\Theta_{1}, \Theta_{2}, \cdots$ the sequence of approximation coefficients of Minkowski's diagonal continued fraction expansion of a real irrational number $x$. For almost all $x$ this is a uniformly distributed sequence in the interval $\left[0, \frac{1}{2}\right]$. The average distance between two consecutive terms of this sequence and their correlation coefficient are explicitly calculated and it is shown why these two values are close to $1 / 6$ and 0 , respectively, the corresponding values for a random sequence in $\left[0, \frac{1}{2}\right]$.
\end{abstract}

\section{Communicated by Cor Kraaikamp}

Let $x$ be a real irrational number and $p_{n} / q_{n}, n=1,2, \cdots$ its sequence of regular continued fraction convergents.

The sequence $\theta_{n}, n=1,2, \cdots$ of approximation coefficients of $x$, defined by

$$
\theta_{n}=q_{n}^{2}\left|x-\frac{p_{n}}{q_{n}}\right|, n=1,2, \cdots
$$

is always a sequence in the unit interval. The subsequence of (1), consisting of all elements $\theta_{n}$ with $\theta_{n}<\frac{1}{2}$, is denoted here by

$$
\Theta_{n}, n=1,2, \cdots
$$

This is the sequence of approximation coefficients of Minkowski's diagonal continued fraction, see [5] and [6. It is for almost all $x$ uniformly distributed in the interval $\left[0, \frac{1}{2}\right]$, which follows from the Doeblin-Lenstra conjecture proved in [1] or, without ergodic theoretical methods, from a result of $\mathrm{Erdös}$, 2], as pointed out by $\mathrm{It} \mathrm{o}$ and $\mathrm{Nakada}$ in $[3]$.

2010 Mathematics Subject Classification: 11K50.

Keywords: Continued fractions, approximation coefficients, metrical theory. 
Reading the paper [7 by Pillichshammer and Steinerberger on the average distance between consecutive terms of uniformly distributed sequences, it occurred to the author that the sequences (2) are also 'typical examples of uniformly distributed sequences' and he wondered what the average distance or expectation

$$
\mathcal{E}\left(\left|\Theta_{n+1}-\Theta_{n}\right|\right)=\lim _{N \rightarrow \infty} \frac{1}{N} \sum_{n=1}^{N}\left|\Theta_{n+1}-\Theta_{n}\right|
$$

might be for them.

The answer turned out to be an explicitly calculable constant with a numerical value $0.164017 \cdots$, for almost all $x$. This is close to $\frac{1}{6}$, the value for a random sequence in $\left[0, \frac{1}{2}\right]$, as remarked by Professor Steinerberger to whom the author mentioned the result. This remark motivated the calculation of the correlation between two consecutive elements of (2). It yielded, for almost all $x$, the value $0.019290 \cdots$ which is again close to the corresponding value for a random sequence, namely 0 . More precisely, we shall prove the following theorem.

Theorem. Denote by

$$
\Theta_{1}, \Theta_{2}, \cdots
$$

the sequence of approximation coefficients of Minkowski's diagonal continued fraction expansion of a real irrational number $x$.

By definition this is always a sequence in $\left[0, \frac{1}{2}\right]$. For almost all $x$ these sequences show the following three similarities with a random sequence in $\left[0, \frac{1}{2}\right]$ :

1. They are uniformly distributed in the interval $\left[0, \frac{1}{2}\right]$,

2. The average distance between two consecutive terms is close to $\frac{1}{6}$, namely,

$$
\lim _{N \rightarrow \infty} \frac{1}{N} \sum_{n=1}^{N}\left|\Theta_{n+1}-\Theta_{n}\right|=\frac{25}{24}+\log 2-\frac{\pi}{2}=0.164017 \cdots,
$$

3. The correlation coefficient $\mathcal{R}\left(\Theta_{n}, \Theta_{n+1}\right)$ between two consecutive terms is almost 0 , namely,

$$
\mathcal{R}\left(\Theta_{n}, \Theta_{n+1}\right)=\frac{111}{5}-32 \log 2=0.019290 \cdots
$$

Proof of the theorem.

1. Where to find a proof of part 1 of the theorem was already mentioned.

2. For a proof of part 2 of the theorem we turn to the fundamental paper by K r a a i k a m p, 5], on Minkowski's expansion. The casual remark on page 207: "In a similar way one could determine the distribution of the sequence $\left(\Theta_{k-1}-\Theta_{k}\right)_{k \geq 0}$ on the interval $\left[-\frac{1}{2}, \frac{1}{2}\right] "$ tells one how this mean value can be calculated. 


\section{A NOTE ON THE CONTINUED FRACTION OF MINKOWSKI}

Let $0<z<\frac{1}{2}$. Denote by $\Omega_{1}(z)$ that part of the first quadrant of the $(x, y)$ -plane for which

$$
0<x<\frac{1}{2}, \quad y<x, \quad y>x-z,
$$

and by $\Omega_{2}(z)$ that part of $\Omega_{1}(z)$ which lies below the parabola

$$
(x-y)^{2}+x+y=\frac{3}{4} \text {. }
$$

We now apply Kraaikamp's Theorem 4.2: Define $F_{1}$ by

$$
F_{1}(z)=4 \iint_{\Omega_{1}(z)} \frac{\mathrm{d} x \mathrm{~d} y}{\sqrt{1-4 x y}}
$$

and $F_{2}$ by

$$
F_{2}(z)=4 \iint_{\Omega_{2}(z)} \frac{\mathrm{d} x \mathrm{~d} y}{\sqrt{1+4 x y}}
$$

Then

$$
F=F_{1}+F_{2}
$$

is the distribution function, for almost all $x$, of the sequence

$$
\left|\Theta_{n+1}-\Theta_{n}\right|, n=1,2, \cdots
$$

We attack both integrals with the substitution

$$
\begin{aligned}
\xi & =x+y, \\
\eta & =x-y
\end{aligned}
$$

which has a determinant of Jacobi equal to -2 . We then find that

$$
F_{1}(z)=2 \iint_{\Omega_{1}^{*}(z)} \frac{\mathrm{d} \xi \mathrm{d} \eta}{\sqrt{1-\xi^{2}+\eta^{2}}}
$$

and

$$
F_{2}(z)=2 \iint_{\Omega_{2}^{*}(z)} \frac{\mathrm{d} \xi \mathrm{d} \eta}{\sqrt{1+\xi^{2}-\eta^{2}}}
$$

where $\Omega_{1}^{*}(z)$ and $\Omega_{2}^{*}(z)$ are the images of $\Omega_{1}(z)$ and $\Omega_{2}(z)$. Hence

$$
F_{1}(z)=2 \int_{0}^{z}\left(\int_{\xi=\eta}^{\xi=1-\eta} \frac{\mathrm{d} \xi}{\sqrt{1-\xi^{2}+\eta^{2}}}\right) \mathrm{d} \eta=2 \int_{0}^{z}\left(\arctan \frac{1-\eta}{\sqrt{2 \eta}}-\arctan \eta\right) \mathrm{d} \eta
$$

from which we see that

$$
F_{1}^{\prime}(z)=2\left(\arctan \frac{1-z}{\sqrt{2 z}}-\arctan z\right)
$$

and from which it follows that 


$$
F_{1}(z)=2\left(\sqrt{2 z}+\log (1+z-\sqrt{2 z})-z \arctan z+z \arctan \frac{1-z}{\sqrt{2 z}}\right) .
$$

In a similar way one finds

and

$$
F_{2}^{\prime}(z)=2(\log 2+\log (1-z))
$$

$$
F_{2}(z)=2(z \log 2-z-(1-z) \log (1-z)) .
$$

Thus we have obtained an explicit expression for the distribution function $F$ from (4) on the interval $\left[0, \frac{1}{2}\right]$.

Now the rather complicated function $F$ is on this interval surprisingly well approximated by the parabola $G$

$$
G(z)=-4 z^{2}+4 z .
$$

If one plots both graphs on an 27-inch computer screen, one hardly sees the difference. On $\left[0, \frac{1}{2}\right]$ one has

$$
0<F(z)-G(z)<0.012
$$

and on $\left[\frac{1}{4}, \frac{1}{2}\right]$ the difference is even smaller than 0.006 .

Let

$$
\xi_{1}, \xi_{2}, \xi_{3}, \cdots
$$

be a hypothetical sequence, uniformly distributed in the interval $\left[0, \frac{1}{2}\right]$, for which the sequence

$$
\left|\xi_{2}-\xi_{1}\right|,\left|\xi_{3}-\xi_{2}\right|,\left|\xi_{4}-\xi_{3}\right|, \cdots,
$$

has the $G$ from (7) as distribution function. Then

Further

$$
\mathcal{E}\left(\left|\xi_{n+1}-\xi_{n}\right|\right)=\frac{1}{6}
$$

and from this and from

$$
\mathcal{E}\left(\left(\xi_{n+1}-\xi_{n}\right)^{2}\right)=\frac{1}{24},
$$

it follows that

$$
\mathcal{E}\left(\xi_{n}^{2}\right)=\frac{1}{12}
$$

So

$$
\mathcal{E}\left(\xi_{n} \xi_{n+1}\right)=\frac{1}{16} .
$$

$$
\mathcal{E}\left(\xi_{n} \xi_{n+1}\right)=\mathcal{E}\left(\xi_{n}\right) \mathcal{E}\left(\xi_{n+1}\right),
$$

consequently the correlation coefficient between $\xi_{n}$ and $\xi_{n+1}$, denoted as

$$
\mathcal{R}\left(\xi_{n}, \xi_{n+1}\right), \quad \text { is } 0 .
$$




\section{A NOTE ON THE CONTINUED FRACTION OF MINKOWSKI}

Therefore one may expect that the limit (3) is close to $\frac{1}{6}$ and that the correlation

$$
\mathcal{R}\left(\Theta_{n}, \Theta_{n+1}\right)
$$

between two consecutive approximation coefficients of Minkowski's continued fraction is almost 0 .

After the observation of the similarity between the obtained distribution function $F$ and a parabola and of some properties of a hypothetical sequence with this parabola as distribution function for the distances of consecutive terms, we finish the proof of part 2 of the theorem.

The first moment of the distribution $F$ equals

$$
2 \int_{0}^{\frac{1}{2}} z\left(\arctan \frac{1-z}{\sqrt{2 z}}-\arctan z\right) \mathrm{d} z+2 \int_{0}^{\frac{1}{2}} z(\log 2+\log (1-z)) \mathrm{d} z .
$$

These two integrals are

$$
\frac{5}{3}-\frac{\pi}{2} \quad \text { and } \quad \log 2-\frac{5}{8}, \quad \text { respectively, }
$$

which proves part 2 of the theorem.

3. For a proof of part 3 of the theorem we need to calculate

$$
\mathcal{E}\left(\left(\Theta_{n+1}-\Theta_{n}\right)^{2}\right)=\int_{0}^{\frac{1}{4}} z \frac{\mathrm{d}}{\mathrm{d} z} F(\sqrt{z}) \mathrm{d} z .
$$

Elementary but tedious calculations show that this integral equals

$$
-\frac{53}{60}+\frac{4}{3} \log 2 \text {. }
$$

The reader may verify this with Mathematica (details are also available, on request via e-mail, from the author).

It follows that

which then yields

$$
\mathcal{E}\left(\Theta_{n} \Theta_{n+1}\right)=\frac{21}{40}-\frac{2}{3} \log 2
$$

$$
\mathcal{R}\left(\Theta_{n}, \Theta_{n+1}\right)=\frac{111}{5}-32 \log 2=0.019290 \cdots,
$$

positive but, as expected, close to 0 .

In 4] approximation coefficients connected with the nearest mediants are added to the sequence (1) which yields in a natural way a uniformly distributed sequence in $[0,1]$. It would be very interesting to find corresponding results for this sequence, in particular to see whether it is also very close, perhaps even closer, to the hypothetical series (86). 


\section{HENDRIK JAGER}

\section{REFERENCES}

[1] BOSMA, W.-JAGER, H.-WIEDIJK, F.: Some metrical observations on the approximation by continued fractions, Indag. Math. Series A 86, 1983, 281-299.

[2] ERDÖS, P.: Some results on Diophantine approximation, Acta Arith. 5 (1959), 359-369.

[3] ITO, SH.-NAKADA, H.: On natural extensions of transformations related to Diophantine approximations, In: Proceedings of the Conference on Number Theory and Combinatorics, Japan 1984 (Tokyo, Okayama and Kyoto, 1984), World Sci. Publ. Co., Singapore, 1985. pp. 185-207.

[4] JAGER, H.: Some metrical observations on the approximation of an irrational number by its nearest mediants, Period. Math. Hungar. 23 (1991), no. 1, 5-16.

[5] KRAAIKAMP, C.: Statistic and ergodic properties of Minkowski's Diagonal Continued Fraction, Theoretical Comp. Sc. 65 (1989), 197-212.

[6] PERron, O.: Die Lehre von den Kettenbrüchen, Band I, B. G. Teubner-Stuttgart, 1977.

[7] PILLICHSHAMMER, F.-STEINERBERGER, S.: Average distance between consecutive points of uniformly distributed sequences, Unif. Distrib. Theory 4 (2009), no. 1, 51-67.

Received October 10, 2016

Accepted February 15, 2017

\author{
Hendrik Jager \\ Oude Larenseweg 26 \\ 7214 PC Epse \\ THE NETHERLANDS
}

E-mail: epserbos@upcmail.nl 